\title{
Perioperative Management of Pulmonary Hypertension in Pediatric Cardiac Surgery
}

\author{
Nabil A Mageed, Ibrahim I Abd El Baser* and Hani I Taman \\ Department of Anesthesia and Surgical Intensive Care, Faculty of Medicine, Mansoura University, Egypt
}

*Corresponding author: Ibrahim I Abd El Baser, Department of Anesthesia and

Surgical Intensive Care, Faculty of Medicine, Mansoura University, Egypt.

Received Date: March 11, 2020

Published Date: March 17, 2020

\begin{abstract}
Pediatric pulmonary hypertension (PH) is associated with many cardiovascular, and pulmonary diseases that result in a high risk of morbidity and mortality. The cause of PH in children is mainly due to congenital heart disease (CHD). The proper preoperative evaluation and the accurate anesthetic management are essential to obtain the best outcome. The perioperative anesthetic goal is directed to avoid pulmonary hypertensive crisis which is associated with poor outcome in children with PH secondary to CHD undergoing cardiopulmonary bypass. The purpose of this review is to discuss the pathophysiology of PH in children with $\mathrm{CHD}$ and to show how to avoid and manage the perioperative pulmonary hypertensive crisis.

Keywords: Pulmonary hypertension; Congenital heart diseases; Pulmonary hypertensive crisis; Cardiac surgery; Pulmonary vasodilators
\end{abstract}

\section{Definition and Clinical Classification}

\section{Definition}

Pulmonary hypertension $(\mathrm{PH})$ is confirmed only after 3 months from birth as during the fetal circulation the pulmonary artery pressure (PAP) is equal to the systemic arterial pressure. Pulmonary hypertension is confirmed when the mean pulmonary arterial pressure is greater than $25 \mathrm{~mm} \mathrm{Hg}$ [1].

\section{Clinical classification}

Clinical classification of $\mathrm{PH}$ associated with congenital heart disease (CHD) (modified from Simonneau G, et al. [2]):

a. Eisenmenger syndrome which starts as left to right shunts that are associated with large intra- and extra-cardiac defects then progress with time to marked rise in pulmonary vascular resistance (PVR) and shunt reversal (right to left), cyanosis and polycythemia are features.

b. Left-to-right shunts that are associated with moderate to large cardiac defects that are accompanied with mild to moderate elevation in PVR, cyanosis is not present. c. Pulmonary hypertension with significant increase in PVR despite of the presence of small cardiac defects.

d. Post-operative PH, congenital cardiac defect is corrected but $\mathrm{PH}$ persists.

\section{Pathophysiology of Pediatric Ph Due to CHD}

Pulmonary hypertension in CHD is associated with intra or extra cardiac defects, the commonest are atrial septal defect (ASD), ventricular septal defect (VSD) and persistent patent ductus arteriosus that cause left-to-right shunt. Left side cardiac obstructive disease results in postcapillary hypertension [3]. The size of the cardiac defect determines the development of PH [4]. More complex lesions such as common atrioventricular canal defects or truncus arteriosus usually develop PH at very young age. Moreover, the child may develop PH after surgical repair of cardiac defect.

For large, atrial or ventricular septal defects, the difference between systemic and pulmonary vascular resistance will 
determine the degree and direction of the shunt [4]. Patients with a large ASD may develop right-to-left shunt if the pulmonary artery pressure (PAP) become equal or greater than the systemic arterial pressure. Large atrial connections usually develop Eisenmenger syndrome more later in life than other defects [5,6].

Atrial septal defects are pre-tricuspid low-pressure left-toright shunts that result in right ventricular volume overload with subsequent pulmonary circulation overflow, but the low-pressure pulmonary circulation does not result in early elevation in PAP. The size of the ASD and the right ventricular compliance affect the risk of developing $\mathrm{PH}$ [7].

Ventricular septal defects are post-tricuspid cardiac defects with left-to-right shunts at high pressure that are associated with left ventricular volume overload with subsequent congestion of pulmonary circulation. The onset of PAH occurs early during infancy. Without intervention, all patients will develop reversal of shunt when the pulmonary vascular resistance exceeds the systemic vascular resistance with Eisenmenger complex. The pathophysiology of PAH in Eisenmenger is attributed to pulmonary vasoconstriction, hypertrophy of the medial wall and pulmonary vascular bed remodeling [7].

The high pulmonary flow and pressure results in damage of pulmonary vascular endothelium with the breakdown of endothelial barrier. The pulmonary vascular endothelial dysfunction results in an increase in the level of pulmonary vasoconstrictors (thromboxane and endothelin-1) and decrease in pulmonary vascular vasodilators (nitric oxide, prostaglandin I2 and vasoactive peptide) [7]

\section{Preoperative Evaluation}

Prior to a procedure, the anesthesiologist must ensure that the child's PH status is optimized (not experiencing acute $\mathrm{PH}$ exacerbation), has no acute comorbidity that might worsen PH (infection, reactive airway exacerbation, gastrointestinal illness with dehydration), and all PH medications are taken per schedule. Children with moderate, severe, or poorly responsive $\mathrm{PH}$ deserve preoperative multidisciplinary planning and aggressive pharmacologic optimization prior to surgery. Strong consideration should be given to preoperative overnight intravenous hydration during fasting in children at higher perioperative risk.

Chest X-ray may indicate enlargement of main pulmonary artery and/or the right ventricle. Transthoracic Doppler echocardiography (TTE) can determine the systolic pulmonary artery pressure, pulmonary vascular resistance, size and function of cardiac chambers and the type of cardiac lesion. Right heart catheterization to measure pulmonary artery pressures, PVR and cardiac output. Acute reactivity of pulmonary vascular bed may also be examined [8].

\section{Preoperative Management}

\section{General measures}

Early intervention either by catheterization or surgery to correct the congenital cardiac defect. Avoidance of dehydration and exercise. Children with Eisenmenger's syndrome are at increased risk of coagulopathy and high incidence of thrombosis of pulmonary artery but the prophylactic anti coagulation is not recommended.

\section{Pharmacological treatment}

The aim of the use of pulmonary vasodilators is to control the high pulmonary artery pressure. This will delay or prevent the progression of the disease and increase the vasoreactivity levels.

Antagonists of Calcium channel: An initial trial of calcium channel antagonists should be carried out in children older than 1 year. Amlodipine has been recommended to be beneficial for Idiopathic PAH. The use of calcium channel antagonists should only be continued in patients who experienced a fall in mean PA pressure and PVR by $20 \%$ [9]. =

Phosphodiesterase inhibitors: Sildenafil is a potent and selective pulmonary vasodilator. Sildenafil acts by inhibiting phodiesterase V (PDE V), which is responsible for the breakdown of cGMP in pulmonary tissue. This results in an increase in cGMP with subsequent calcium-mediated vascular smooth muscle relaxation [10]. Milrinone is a PDE III inhibitor which induces pulmonary vasodilation by its actions through a cyclic adenylate monophosphate (cAMP) mediated signaling pathway [11].

Endothelin receptor antagonists: Bosentan is a non-selective (endothelin receptor $\mathrm{A}$ and $\mathrm{B}$ ) antagonist that decrease the mean PA pressure and PVR, and improve life quality in patients with idiopathic PH. Sitaxsentan and Ambrisentan are new antagonists that have been shown to increase the exercise tolerance in patients with PH [12].

Prostacyclin agonists: Intravenous prostacyclins include (epoprostenol, treprostinil). Inhalation route is used for (iloprost) while oral route is used for (beraprost), adverse effects include flushing, jaw pain in the mandible, headaches, skin rashes and platelet dysfunction [13].

\section{Intraoperative Management}

Cardiac surgical patients with $\mathrm{PH}$ have a significant risk for perioperative morbidity and mortality. Pulmonary hypertension patients require full evaluation and management of their disease states for optimal risk reduction, as described earlier. Medications taken chronically for PH should be continued perioperatively [14]. If a patient is unable to continue an oral pulmonary vasodilator in the perioperative period, opportunities to provide pulmonary vasodilators via alternative routes should be pursued [15]. Fasting is required before anesthesia for surgery. However, and especially 
for patients with heart defects, prolonged fasting may lead to dehydration and hypervolemia. Guidelines allow clear fluids up to 2 hours preoperative, Breast milk up to 4 hours, while formula milk and solid foods are permitted 6-8 hours preoperative. Critical ill patients fasting should be tailored to keep them in optimal conditions preoperatively with iv fluids [15].

The overarching goal of anesthetic management is to provide adequate anesthesia and analgesia, while avoiding RV failure, increased PVR, low SVR, and coronary ischemia. There is currently no reported "best" anesthetic that achieves these goals. Given the limited cardiopulmonary reserve of many of these children, the operating room should be carefully prepared before beginning anesthesia. All equipment and medications that may be required should be immediately accessible, including those for emergent resuscitation. An iNO machine on standby in the OR is encouraged to rescue a $\mathrm{PH}$ crisis in high-risk children. Any continuously administered pulmonary vasodilator (inhaled or intravenous) must be maintained, and a central venous line for infusion of continuous prostacyclin analogs should be considered a dedicated line and never used for intraoperative venous access. All oral PH medications should be taken on schedule with a small sip of water The decision to premedicate the child with PAH carries risks and benefits. Premedicating an anxious child can alleviate the increased PVR and oxygen consumption associated with agitation preinduction; however, over sedation and resultant hypoventilation and hypercapnia should be avoided, especially in children with baseline airway obstruction. Use of pulse oximetry is essential after premedication [16].

\section{Induction of anesthesia}

Events known to exacerbate elevation in PVR (i.e, hypoxia, hypercarbia, acidosis, hypothermia, and sympathetic stimulation) should be avoided [14]. Before induction of anesthesia the patient must be attached to pulse oximetry and 5 leads electrocardiography (ECG). It's the period of maximum risk as it's the time of potential hypoxia, hypercapnia, hypotension from induction agents, and noxious stimulus during airway instrumentation. The goal is an induction with minimal changes to SVR and PVR, while ensuring adequate ventilation. Preparation for intubation requires a balance between sufficient analgesia to blunt the noxious stimulus while also maintaining adequate SVR and myocardial performance. The trachea should be intubated quickly without allowing significant hypoxia and hypercapnia. After preoxygenation, the induction of general anesthesia can be performed using small, titrated doses of an induction agent, fentanyl, and a muscle relaxant. In patients with a severely depressed LV ejection fraction, a technique based on midazolam and fentanyl may be indicated to avoid cardiac depression. Opioids blunt the response to noxious stimuli and as such limit the risk of PH crisis from that provocation. Ketamine has been shown to have little to no pulmonary vascular effects [17]. Etomidate is associated with sable hemodynamics. Propofol on the other hand causes marked reduction in SVR and has a more limited role in critically ill patients $[17,18]$.

\section{Monitoring}

In addition to the standard American Society of Anesthesiologists monitors, patients with PH presenting for cardiac surgical procedures should have an arterial catheter, central venous introducer, and pulmonary artery catheter [19]. Simultaneous monitoring of systemic and pulmonary pressures permits the early detection of PAP elevation, in particular those times when PAP may approach or exceed the mean arterial pressure (MAP), indicating the need for intensive pulmonary vasodilator therapy. Intraoperative transesophageal echocardiography (TEE) should be used to guide inotropic and fluid therapy.

\section{Maintenance of anesthesia}

Systemic vascular resistance should be maintained and aggressively treated with systemic vasoconstrictors because decreased coronary perfusion to a hypertrophied right ventricle can progress rapidly to ischemia and hemodynamic collapse. Echocardiography is useful and potentially vital to troubleshooting hemodynamic instability because low systemic pressures can be the result of decreased SVR or RHF $[14,20]$. General anesthesia can be maintained with either intravenous or volatile anesthetics because there has been no determination of clinical benefit to favor one technique over the other [21]. Because most anesthetics impact SVR and myocardial contractility, a balanced technique with narcotic administration to blunt sympathetic stimulation can achieve the desired hemodynamic goals [19]. Inhaled anesthetics promote peripheral vasodilation, and a limited amount of this effect can prove beneficial in decreasing RV preload and PAP [19]. Continuous monitoring for RV decompensation with TEE is essential, and RV decompensation should be treated aggressively to prevent further rapid deterioration [22]. In the pre- and postCPB periods, continuous attention should be paid to oxygenation, ventilation, acid-base and volume status, and anesthetic depth to avoid factors that can increase PAP.

In situations of hemodynamic instability in the prebypass period, there may be a need for pharmacologic intervention with intravenous or inhalation agents to decrease PVR, increase cardiac output, and maintain SVR. A common combination to meet these hemodynamic goals is milrinone with epinephrine. Inotropes with vasodilating properties at times can decrease coronary perfusion, create RV ischemia and dysfunction, and worsen cardiac output rather than improving it $[14,19]$. Vasopressin can be added to maintain systemic pressure in the event that vasodilation is poorly tolerated. The treatment for increased PVR and/or RV failure with decreased systemic pressures should include therapy more specific to the pulmonary vascular bed, such as inhaled vasodilators NO, iloprost, or epoprostenol [14,19]. 


\section{Pulmonary hypertensive crisis}

Diagnosis and pathophysiology: Pulmonary hypertensive crisis is defined by an acute elevation in pulmonary artery pressure with increase the ratio of mean PAP to mean systemic arterial pressure more than 0.75 . This is associated with an increase in central venous pressure by $20 \%$ or more, a drop in mean arterial systemic pressure by $20 \%$ or more, a fall in arterial oxygen saturation less than $90 \%$, and clinical evidence of low cardiac output syndrome [23]. A PH crisis occurs when there is an acute insult to baseline cardiopulmonary mechanics (e.g., rise in PVR), compensatory mechanisms of chronic PH fail, RV function decompensates, and LV preload acutely decreases resulting in inadequate cardiac output and coronary perfusion. This cycle of deterioration, as shown in Figure 1, can quickly lead to biventricular ischemia and cardiac arrest [16]. Hypoxia, hypercapnia, acidosis, hypothermia, and noxious stimuli increase PVR and are known causes of acute PH crisis under anesthesia, particularly in children who retain modifiable PVR. A precipitous fall in SVR during induction or maintenance of anesthesia also risks increasing the PAP/systemic arterial pressure ratio and provoking $\mathrm{PH}$ crisis. Other mechanism may instigate a PH crisis, especially in children with advanced PAH and relatively fixed PVR. These include reduced preload with subsequently reduced RV output, anesthesia-induced myocardial depression, coronary hypoperfusion and ischemia, arrhythmias, pneumothorax, pulmonary embolism, sepsis, pericardial effusion, bronchospasm and anaphylaxis.

After weaning from cardiopulmonary bypass, patients are at high risk for the development of $\mathrm{PH}$ crisis which is a life threatening condition. Endothelial cell damage is exacerbated by hypothermia, cardioplegia and the non pulsetile flow of cardiopulmonary bypass $[24,25]$. Recently, the incidence of PH crisis is significantly reduced by improvement of surgical techniques that is associated with reduced duration of cardiopulmonary bypass, advanced myocardial protection strategies and modified ultrafiltration. Blood flow from the bronchial arteries prevents pulmonary infarction during cardiopulmonary bypass but is not sufficient to prevent pulmonary vascular endothelial damage $[25,26]$. After weaning from cardiopulmonary bypass, a further damage of endothelium may occur by reperfusion injury (Figure 1).

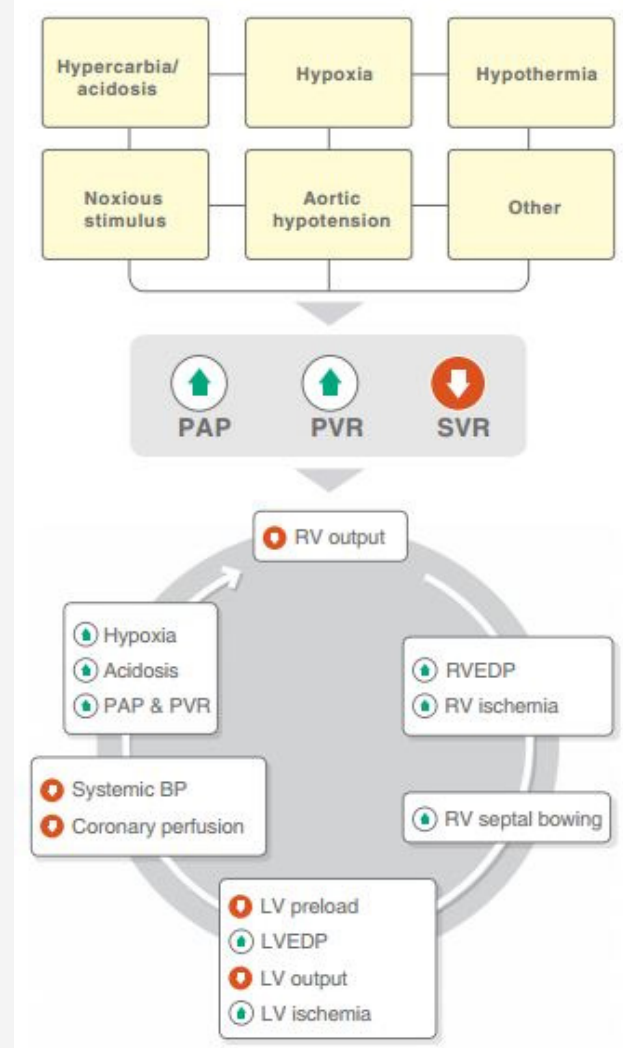

Figure 1 : The pathophysiology of pulmonary hypertensive crisis [16]

Pulmonary hypertensive crisis is accurately defined by the use of a PA catheter that measure PA pressure directly. The clinical signs of a PH crisis vary from gradual decrease in arterial oxygen saturation and cardiac output to abrupt life threatening cardiopulmonary collapse. During anesthesia, the signs of PH crisis include decreased arterial saturation, decreased endtidal $\mathrm{CO}_{2}$, sinus tachycardia initially that is often followed by bradycardia, ECG changes indicating right ventricular strain, and hypotension.

\section{Management of PH crisis}

If a PH crisis occurs, immediate treatment includes administration of $100 \%$ oxygen, mild hyperventilation, 
administration of a selective pulmonary vasodilator (iNO), ventricular support (epinephrine, vasopressin), systemic alkalinization(sodium bicarbonate and/or hyperventilation), and deepening of anesthesia if noxious stimuli are a possible trigger $[27,28]$. RV function is preload dependent in pediatric $\mathrm{PH}$, and a fluid bolus may be beneficial if hypovolemia is suspected; however, conditions may worsen in the setting of decompensated RV failure [28]. Administration of pulmonary vasodilators should be considered during a crisis, understanding that their efficacy may be notably less in children with long-standing PAH and nonreactive pulmonary vasculature. iNO is the first choice in mechanically ventilated patients. iNO may be rapidly delivered, and it selectively reduces PVR without a significant decrease in SVR. Many other pulmonary vasodilators (sildenafil, milrinone, prostanoids) also undesirably decrease SVR. Along with the obvious benefit of organ perfusion, maintenance of SVR is important for coronary perfusion and interventricular septal positioning in the setting of acute RV strain. As such, vasopressin may be a useful adjunct during PH crisis. Vasopressin and terlipressin increase SVR while also decreasing PVR, thus beneficially decreasing the PVR:SVR ratio; this is in contrast to phenylephrine and norepinephrine, which increase PVR $[27,29]$.

\section{Weaning From CPB}

Successful separation from CPB is ensured by adhering to the same anesthetic goals as in the prebypass period including the optimization of preload, PVR, SVR, and contractility. In addition, the anticipated effects of the surgery and CPB on PAP and right heart function should be considered when preparing for separation from CPB. Inotropic and vasodilator support should be provided after adequate reperfusion and optimized before separation from CPB. Many combinations can be effective, but a common combination is milrinone with epinephrine to initially separate. Careful attention to hemodynamics with simultaneous monitoring of right heart function with TEE should continue until stability is achieved. Generally, titration of milrinone and epinephrine can target the balance of RV contractility and PAP. The addition of vasopressin can be considered if low SVR and reduced coronary perfusion are of concern. If hemodynamics deteriorates beyond manageability with intravenous therapy, inhaled pulmonary vasodilator therapy is available [30].

The use of right ventricular assisted device may be beneficial if the weaning from cardiopulmonary bypass is difficult Extracorporeal Membrane Oxygenation (ECMO) can be used to stabilize the patient pre-operatively, in case of failure of weaning from cardiopulmonary bypass, or postoperative low cardiac output syndrome. ECMO is utilized in $1.4-5 \%$ of operations for CHD [3133].

\section{Postoperative Care}

In the intensive care unit, the post-operative management is directed mainly to prevent PH crisis. Hypoxia, hpercapnia, acidosis, pain, and hypothermia should be avoided. The patients should be kept sedated and ventilated to correct and avoid any event that may provoke the PH crisis. Pulmonary vasodilators by inhalation or the intravenous route may be used. Patients with PH crisis and right ventricular failure not responding to medical treatment may require Extracorporeal Membrane Oxygenation.

\section{Conclusion}

Perioperative management of the pediatric patient with $\mathrm{PH}$ undergoing open heart surgery is a complex and critical condition. The anesthetic management is aiming to prevent pulmonary hypertensive crisis, which may lead to acute and fatal RV failure.

\section{Acknowledgement}

None.

\section{Conflict of Interest}

No conflict of interest.

\section{References}

1. Debra Boyer D, Pittman JE, Jamie FD, Arunachalam A, Dweik RA, et al. (2016) Pediatric Pulmonary Hypertension. Ann Am Thorac Soc 13(6): 967-969.

2. Simonneau G, Gatzoulis MA, Adatia I, Celermajer D, Denton C, et al. (2013) Updated clinical classification of pulmonary hypertension. J Am Coll Cardiol 62(25): D34-D41.

3. Diller GP, Kempny A, Inuzuka R, Radke R, Wort SJ, et al. (2014) Survival prospects of treatment naive patients with Eisenmenger: a systematic review of the literature and report of own experience. Heart 100(17): 1366-1372.

4. Simonneau G, Galie N, Rubin LJ, Langleben D, Seeger W, et al. (2004) Clinical classification of pulmonary hypertension. J Am Coll Cardiol 43(Suppl 12): S5 -S12.

5. Farber HW, Loscalzo J (2004) Pulmonary arterial hypertension. N Engl J Med 351(16): 1655-1665.

6. Kozlik Feldmann R, Hansmann G, Bonnet D, Schranz D, Apitz C, et al. (2016) Pulmonary hypertension in children with congenital heart disease (PAH-CHD, PPHVD-CHD). Expert consensus statement on the diagnosis and treatment of paediatric pulmonary hypertension. The European Paediatric Pulmonary Vascular Disease Network endorsed by ISHLT and DGPK. Heart 102(Suppl2): ii42-ii48.

7. Diller GP, Gatzoulis MA (2007) Pulmonary vascular disease in adults with congenital heart disease. Circulation 115(8): 1039-1050.

8. Hoeper M, Bogaard H, Condliffe R, Frantz R, Khanna D, et al. (2013) Definitions and diagnosis of pulmonary hypertension. J Am Coll Cardiol 62(25 Suppl): D42-D50.

9. Mawatari E, Hongo M, Sakai A, Terasawa F, Takahashi M, et al. (2007) Amlodipine prevents monocrotaline-induced pulmonary arterial hypertension and prolongs survival in rats independent of blood pressure lowering. Clin Exp Pharmacol Physiol 34(7): 594-600.

10. Leibovitch L, Matok I, Paret G (2007) Therapeutic applications of sildenafil citrate in the management of paediatric pulmonary hypertension. Drugs 67(1): 57-73.

11. Bassler D, Choong K, Mc Namara P, Kirpalani H (2006) Neonatal persistent pulmonary hypertension treated with milrinone: four case reports. Biol Neonate 89(1): 1-5.

12. Barst RJ, Rich S, Widlitz A, Horn EM, Mc Laughlin V, et al. (2002) Clinical efficacy of sitaxsentan, an endothelin-A receptor antagonist, in patients with pulmonary arterial hypertension: open-label pilot study. Chest 121(6): 1860-1868 
13. Badesch DB, Mclaughlin VV, Delcroix M, Vizza CD, Olschewski H, et al. (2004) Prostanoid therapy for pulmonary arterial hypertension. J Am Coll Cardiol 43(Suppl 12): S56-S61.

14. Pritts CD, Pearl RG (2010) Anesthesia for patients with pulmonary hypertension. Curr Opin Anaesthesiol 23(3): 411-416.

15. Smith I, Kranke P, Murat I, Smith A, O Sullivan G, et al. (2011) Perioperative fasting in adults and children: guidelines from the European Society of Anaesthesiology. Eur J Anaesthesiol 28(8): 556-569.

16. Latham GJ, Yung D (2019) Current understanding and perioperative management of pediatric pulmonary hypertension. Paediatr Anaesth 29(5): 441-456.

17. Friesen RH, Twite MD, Nichols CS, Cardwell KA, Pan Z, et al. (2016) Hemodynamic response to ketamine in children with pulmonary hypertension. Pediatr Anesth 26(1): 102-108.

18. Twite MD, Friesen RH (2013) The anesthetic management of children with pulmonary hypertension in the cardiac catheterization laboratory. Anesthesiol Clin 32(1): 157-173.

19. Fox C, Kalarickal PL, Yarborough MJ, Jin JY (2008) Perioperative management including new pharmacological vistas for patients with pulmonary hypertension for noncardiac surgery. Curr Opin Anaesthesiol 21(4): 467-472.

20. Ross AF, Ueda K (2010) Pulmonary hypertension in thoracic surgical patients. Curr Opin Anaesthesiol 23(1): 25-33

21. Lai HC, Lai HC, Wang KY, Lee WL, Ting CT, et al. (2007) Severe pulmonary hypertension complicates postoperative outcome of non-cardiac surgery. Br J Anaesth 99(2): 184-190.

22. Teo YW, Greenleigh DL (1991) Update on anaesthetic approach to pulmonary hypertension. Eur J Anaesthesiol 27(4): 317-323.

23. Hopkins RA, Bull C, Haworth SG, De Leval MR, Stark J (1991) Pulmonary hypertensive crises following surgery for congenital heart defects in young children. Eur J Cardiothorac Surg 5(12): 628-634.

24. Wessel DL, Adatia I, Giglia TM, Thompson JE, Kulik TJ (1993) Use of inhaled nitric oxide and acetylcholine in the evaluation of pulmonary hypertension and endothelial function after cardiopulmonary bypass. Circulation (5 Pt 1): 2128-2138.

25. Lesage AM, Tsuchioka H, Young WG Jr, Sealy WC (1966) Pathogenesis of pulmonary damage during extracorporeal perfusion. Arch Surg 93(6): 1002-1008.

26. Anyanwu E, Dittrich H, Gieseking R, Enders HJ (1982) Ultrastructural changes in the human lung following cardiopulmonary bypass. Basic Res Cardiol 77(3): 309-322.

27. Kaestner M, Schranz D, Warnecke G, Apitz C, Hansmann G, et al. (2016) Pulmonary hypertension in the intensive care unit. Expert consensus statement on the diagnosis and treatment of paediatric pulmonary hypertension. The European Paediatric Pulmonary Vascular Disease Network endorsed by ISHLT and DGPK. Heart 102(Suppl 2): ii57-ii66.

28. Friesen RH, Williams GD (2008) Anesthetic management of children with pulmonary arterial hypertension. Pediatr Anesth 18: 208-216.

29. Siehr SL, Feinstein JA, Yang W, Peng LF, Ogawa MT, et al. (2016) Hemodynamic effects of phenylephrine, vasopressin, and epinephrine in children with pulmonary hypertension: a pilot study. Pediatr Crit Care Med 17(5): 428-437.

30. Thunberg C, Gaitan D, Grewal A, Ramakrishna H, Stansbury LG, et al. (2013) Pulmonary Hypertension in Patients Undergoing Cardiac Surgery: Pathophysiology, Perioperative Management, and Outcomes. Journal of Cardiothoracic and Vascular Anesthesia 27(3): 551-572.

31. Mascio CE, Austin EH, Jacobs JP, Jacobs ML, Wallace AS, et al. (2014) Perioperative mechanical circulatory support in children: an analysis of the Society of Thoracic Surgeons Congenital Heart Surgery Database. J Thorac Cardiovasc Surg 147(2): 658 -664.

32. Salvin JW, Laussen PC, Thiagarajan RR (2008) Extracorporeal membrane oxygenation for postcardiotomy mechanical cardiovascular support in children with congenital heart disease. Paediatr Anaesth 18(12): 11571162.

33. Sasaki T, Asou T, Takeda Y, Onakatomi Y, Tominaga T, et al. (2014) Extracorporeal life support after cardiac surgery in children: outcomes from a single institution. Artif Organs 38(1): $34-40$. 